\title{
Predicting negative ureteroscopy for stone disease - Minimizing risk and cost
}

\author{
Miguel Eliseu, Roberto Jarimba, Pedro Moreira, Pedro Simões, Paulo Temido, Arnaldo Figueiredo \\ Urology and Renal Transplantation Department, Coimbra Hospital and University Center, Coimbra, Portugal.
}

\begin{abstract}
Summary Introduction: Urolithiasis is common worldwide, with ureteric stones being a particular burden. Ureteroscopy (URS) is one of the most useful procedures in treating ureteric stones not passed spontaneously; this procedure has a complication risk of $4 \%$. Negative URS, with described rates up to $15 \%$, represents an avoidable patient risk and use of medical resources.

Objectives: To describe rates and identify predictive factors for negative URS and to define strategies which would minimize patient and financial burden from these unnecessary procedures. Materials and methods: A retrospective cohort study analyzed patients who underwent URS in our Center to treat ureteric stones over a period of 2 years. Patient age, gender, and comorbidities, as well as laboratory and imaging findings, were analyzed.

Results: 262 patients underwent URS for ureteric stones. The female population was $50.8 \%$ with a mean age of 56.89 years. A total of 78 (29.8\%) URS procedures were negative. Univariate analysis showed a higher prevalence of negative URS in female patients, as well as in primary, smaller, and radiolucent stones. At multivariate analysis, a logistic regression model correctly classified $76 \%$ of patients, with smaller stone size and radiolucency being significant predictors of negative URS. Discussion and conclusions: Our Center showed a high rate of negative URS, higher than commonly described in the literature. Female patients tend to have an even higher rate, possibly due to unnoticed passage of stones. Patients with small, radiolucent stones showed the highest rates of negative URS.
\end{abstract}

KEY WORDS: Urolithiasis; Ureteric; Stone; Ureteroscopy; Negative.

Submitted 2 January 2021; Accepted 2 July 2021

\section{INTRODUCTION}

Urolithiasis is very common worldwide, with prevalence rates described in general population of 1-20\% (1-4); countries with high standard of life show increasing rates over the past decades, with over 10\% reported prevalence (5-7). Ureteric stones pose a particular burden with frequent need of emergency visits and possible need for admittance and invasive procedures $(7,8)$.

Ureteroscopy (URS) is one of the most useful methods for treating ureteric stones not passed spontaneously (4, 10, 11). This procedure is generally considered safe, involving a complication risk of $4 \%$, with many being performed in an outpatient basis (12-14).

A mean overall cost of $\$ 2801$ per procedure has been described in a systematic review (13).
When a patient is offered a URS for a ureteric stone, the possibility of spontaneous expulsion still exists before the procedure, eventually unnoticed by the patient. If no other measures are taken to detect persistence of stones before surgery, a "stoneless" or "negative" procedure (hence, unnecessary) would be performed (14). Negative URS, with described rates up to $15 \%$, represents a avoidable patient risk and use of medical resources (15-18). Predicting negative URS preoperatively and cancelling the procedure would prevent this unnecessary burden (14).

Several factors predicting negative URS have been studied. Smaller stone size is associated with increased probability of unnoticed expulsion and negative URS $(15,17)$; other reported factors include distal stone positioning (17) and female gender $(15,16)$. Time since last imaging study does not appear to influence stoneless procedure rates (17). Other potential influencing factors showed varying results (15-18). The objective of this paper is to describe rates and identify predictive factors of negative URS and to define strategies which would minimize patient and financial burden of these unnecessary procedures.

\section{Materials AND MEthods}

A retrospective cohort study analyzed all patients who underwent URS in our Center over a period of 2 years. Only procedures to treat ureteric stones were included; all were performed in the same Center, with similar surgical equipment. Several factors were reviewed, including patient age, gender and comorbidities, previous procedures, as well as clinical, laboratory and imaging findings. To comply with the purpose of the study, patients with negative URS were identified, and a potential correlation with the above-mentioned factors was investigated. Patients who had negative URS were followed with ultrasound or computerised tomography (CT), and those with presence of stone suspected of retrograde migration were not included; patients in which complete URS was not feasible were also not included. Statistical analysis was performed using SPSS $23^{\circledR}$, including univariate Mann-Whitney and KruskalWallis tests, and a multivariate logistic regression model.

\section{RESULTS}

During the defined period, 262 patients underwent URS as planned treatment for ureteric stones while meeting the selection criteria. The population was $50.8 \%$ female, 
Table 1.

Patient/stone characteristics and acute episode clinical variables; frequencies represented in percentage of valid results and absolute number of cases excluding missing values in brackets.

\begin{tabular}{|c|c|}
\hline $\begin{array}{l}\text { Patient } \\
\text { and stone characteristics }\end{array}$ & $\begin{array}{l}\text { Frequency in valid \% (n) } \\
\text { excluding missing }\end{array}$ \\
\hline Sex & $\begin{array}{l}50.8 \%(133) \\
49.2 \%(129)\end{array}$ \\
\hline $\begin{array}{c}20-34 \\
35-49 \\
50-64 \\
65-80 \\
80 \text { or more }\end{array}$ & $\begin{array}{c}7.3 \%(19) \\
22.9 \%(60) \\
37 \%(97) \\
24.4 \%(64) \\
8.4 \%(22)\end{array}$ \\
\hline $\begin{array}{c}\text { Primary } \\
\text { Recurrent disease }\end{array}$ & $\begin{array}{c}81.5 \%(203) \\
18.5 \%(46)\end{array}$ \\
\hline $\begin{array}{l}\text { Upper ureter } \\
\text { Mid-ureter } \\
\text { Lower ureter }\end{array}$ & $\begin{array}{c}22.0 \%(52) \\
23.7 \%(56) \\
54.3 \%(128)\end{array}$ \\
\hline $\begin{array}{l}\text { Radiopaque } \\
\text { Radiolucent }\end{array}$ & $\begin{array}{c}86.3 \%(202) \\
13.7 \%(32)\end{array}$ \\
\hline Medical expulsive therapy & $\begin{array}{c}53.8 \%(141) \\
11.5 \%(30)\end{array}$ \\
\hline Perc. nephrostomy in acute episode (performed) & $11.8 \%(30)$ \\
\hline JJ stenting in acute episode & $57.5 \%(146)$ \\
\hline
\end{tabular}

with a mean age of 56.89 years (SD +/- 15.705 years). Patients had a mean stone size of $7.7 \mathrm{~mm}(\mathrm{SD}+/-\mathrm{mm})$; $47.8 \%$ of patients had stones between 5 and $9 \mathrm{~mm}$. According to pre-procedure imaging, the lower ureter was the most common stone location (54.3\%), followed by mid (23.7\%) and upper ureter (22\%). Approximately $86.3 \%$ of stones studied were radiopaque on plain $\mathrm{X}$ ray of Kidney-Ureter-Bladder (KUB). Regarding imaging techniques applied at initial diagnosis, renal ultrasound was most used (99.2\% of patients); KUB X-ray was performed in $90.2 \%$ of cases and CT in $78.6 \%$.

Stenting in the acute setting was performed at physician discretion, with Center policy including best practice guidelines; stenting was performed in cases with associated infection, compromised renal function or long-standing pain (over 14 days) irrespective of planned URS or not. A significant proportion of patients underwent ureteric stenting in the acute setting $(57.5 \%$; $=146)$; of those proposed to URS after stenting, $67.8 \%(\mathrm{n}=99)$ underwent KUB, $15.1 \%(n=22)$ underwent CT, with $2.7 \%(n=4)$ having both exams; 29 patients (19.9\%) had no imaging between stenting and surgery. Cases where no evidence of stones was found were not considered for URS.

The mean time between the acute episode and subsequent URS was 61.8 days (+/- 27.076). In patients who underwent stenting prior to URS, mean time from stenting to surgery was 65.3 days (+/- 28.278). Patient and stone characteristics are displayed in Table 1.

A total of 78 URS procedures were negative for stones, representing 29.8\%. Several factors were investigated in univariate analysis, which showed a higher prevalence of negative URS in female patients ( $p=0.023$ ), as well as in primary $(\mathrm{p}=0.001)$, smaller $(\mathrm{p}=0.010)$, and radiolucent stones $(p=0.035)$. These results are displayed in Table 2 .
Several other factors were analyzed, but not found to be predictors of negative URS (Table 3). Namely, anatomical stone position $(p=0.646)$, mean time between the acute episode and subsequent URS ( $p=0.207)$ and mean time from stenting to surgery $(p=0.614)$ did not appear to influence the risk of negative ureteroscopy. There was also no significant difference between patients who did or did not undergo CT scan (negative URS rates of $28.6 \%$ vs $33.9 \%, \mathrm{p}=0.139$ ).

At multivariate analysis, a logistic regression model correctly classified $76 \%$ of patients, with smaller stone size $(p=0.026)$ and radiolucency $(p=0.011)$ being significant predictors of negative URS, and accounting for $47.7 \%$ of the variance. Each mm increase in stone size, showed an impact on OR for negative URS of 0.815 $(\operatorname{expB}=-0.204)$, while radiopaque stones showed an $\mathrm{OR}$

\section{Table 2.}

Significant variables in univariate analysis with respective rates of negative URS in each subgroup and corresponding $p$ values. Negative URS was more common in females, primary cases, small and radiolucent stones.

\begin{tabular}{|l|c|c|}
\hline Clinical Variables & \% negative URS & p value \\
\hline Primary & $32.5 \%$ & $<0.001$ \\
Recurrence & $8.7 \%$ & \\
\hline Female & $36.1 \%$ & 0.023 \\
Male & $23.3 \%$ & \\
\hline $0-4.9 \mathrm{~mm}$ & $56.3 \%$ & 0.010 \\
$5-9.9 \mathrm{~mm}$ & $33.7 \%$ & \\
$10 \mathrm{~mm}$ or more & $15.5 \%$ & 0.035 \\
\hline Radiopaque & $19.8 \%$ & \\
Radiolucent & $43.8 \%$ & \\
\hline
\end{tabular}

Table 3.

Non-significant variables which did not show influence on the rate of negative URS.

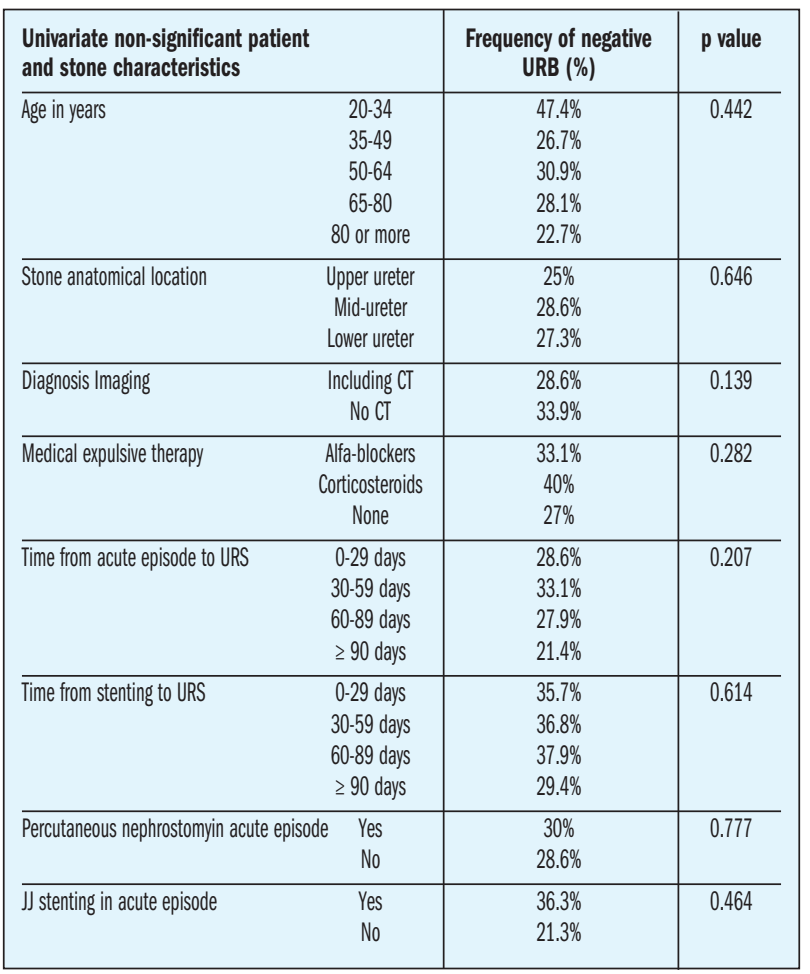




\section{Table 4.}

Logistic regression model accounting for $47.7 \%$ of the variance in the dependent variable (probability of negative URS), correctly classifying $76 \%$ of patients; each $\mathrm{mm}$ increase in stone size with OR of 0.815 and radiopaque stones with OR of 0.240 for negative URS.

\begin{tabular}{|l|c|cc}
\hline Applied variables & Significance (p) & B & $\operatorname{exp~(B)~}$ \\
\hline Sex & 0.234 & \multicolumn{2}{|c}{-} \\
\hline Stone size (absolute value in mm) & 0.026 & -0.204 & 0.815 \\
\hline Primary vs Recurrence & 0.198 & \multicolumn{2}{|c}{-} \\
\hline Radiopacity & 0.011 & -3.716 & 0.240 \\
\hline
\end{tabular}

for negative ureteroscopy of $0.240(\operatorname{expB}=-3.716)$. Table 4 summarises these results.

\section{Discussion AND CONCLUSIONS}

Our Center showed a high rate of negative URS, more than previously described in the literature. A recent systematic review suggests that a publication bias may exist, resulting in series with higher rates of URS not being submitted for peer review, leading to underestimation of its prevalence (19).

Stone position has been described in one previous study as an influencing factor, with distal stones resulting in higher negative URS rates (17); this did not seem to be the case with our population. In accordance with previously reported data (17) time to surgery from last imaging study or stenting also did not influence negative URS rates. Of note, our series presents a significant number of pre-stented patients, more than in previously reported retrospective studies (15) and 19.9\% of those did not undergo further imaging before URS.

Female patients tend to have a higher rate of negative URS, as described in two recent studies $(15,16)$. This is possibly explained by frequent unnoticed passage of stones (due to shorter urethral length and lower voiding pressure) or by a higher frequency of pelvic phleboliths mistaken as ureteric stones (19).

Patients with small, radiolucent stones showed the highest rates of negative URS; this is also in accordance with previously described series $(15,17)$. These patients would benefit the most from pre-operative repeat imaging studies, eventually with non-contrast CT, to identify and preclude unnecessary treatments and costs (14). Prospective studies could help identify more precisely which imaging studies, in which patients and in what timeframe would impact the most in terms of change in planned treatment, to suggest clear guidelines regarding this matter.

\section{REFERENCES}

1. Trinchieri A. Epidemiology of urolithiasis.Arch Ital Urol Androl. 1996; 68:203-49.

2. Trinchieri A. Epidemiology of urolithiasis: an update. Clin Cases Miner Bone Metab. 2008; 5:101-106.

3. Sorokin I, Mamoulakis C, Miyazawa K, et al. Epidemiology of stone disease across the world. World J Urol. 2017; 35:1301-20.

4. Turney BW, Reynard JM, Noble JG, Keoghane SR. Trends in urological stone disease. BJU Int. 2012; 109:1082-7.

5. Rukin NJ, Siddiqui ZA, Chedgy ECP, Somani BK. Trends in upper tract stone disease in England: evidence from the Hospital Episodes Statistics (HES) database. Urol Int. 2017; 98:391-6.

6. Hesse A, Brändle E, Wilbert D, et al. Study on the prevalence and incidence of urolithiasis in Germany comparing the years 1979 vs. 2000. Eur Urol. 2003; 44:709-13.

7. Stamatelou KK, Francis ME, Jones CA, et al. Time trends in reported prevalence of kidney stones in the United States: 19761994. Kidney Int. 2003; 63:1817-23.

8. Kim JW, Kim JY, Ahn ST, et al. Analysis of patients with urolithiasis visiting the emergency department between 2014 and 2016 in Korea: data from the National Emergency Department Information System. Sci Rep. 2019; 9:16630.

9. Gomes J, Vendeira P, Ribau U, Reis M. Urolitíase e cólica renal. Perspectiva terapêtica em Urologia [Urolithiasis and renal colic. Therapeutic approach in urology]. Acta Med Port. 2002; 15:369-80.

10. Geraghty RM, Jones P, Somani BK. Worldwide trends of urinary stone disease treatment over the last two decades: a systematic review. J Endourol. 2017; 31:547-56.

11. Pietropaolo A, Proietti S, Geraghty R, et al. Trends of 'urolithiasis: interventions, simulation, and laser technology' over the last 16 years (2000-2015) as published in the literature (PubMed): a systematic review from European section of Urotechnology (ESUT). World J Urol. 2017; 35:1651-1658.

12. Somani BK, Giusti G, Sun Y, et al. Complications associated with ureterorenoscopy (URS) related to treatment of urolithiasis: the Clinical Research Office of Endourological Society URS Global study. World J Urol. 2017; 35:675-681.

13. Geraghty RM, Jones P, Herrmann TRW, et al. Ureteroscopy is more cost effective than shock wave lithotripsy for stone treatment: systematic review and meta-analysis. World J Urol. 2018; 36:1783-1793.

14. Lloyd P, Johnston T, Coode-Bate J, Keoghane SR. A negative ureteroscopy for stone disease: is it acceptable and is it avoidable? Urol New. 2018; 22(6).

15. Katafigiotis I, Sabler IM, Heifetz EM, et al. "Stoneless" or negative ureteroscopy: a reality in the endourologic routine or avoidable source of frustration? Estimating the risk factors for a negative ureteroscopy. J Endourol. 2018; 32:825-30.

16. Lamberts RW, Conti SL, Leppert JT, Elliott CS. Defining the rate of negative ureteroscopy in the general population treated for upper tract urinary stone disease. J Endourol. 2017; 31:266-71.

17. Kreshover JE, Dickstein RJ, Rowe C, et al. Predictors for negative ureteroscopy in the management of upper urinary tract stone disease. Urology. 2011; 78:748-52.

18. Prattley S, Rice P, Pietropaolo A, et al. Predictors and results of negative ureteroscopy for treatment of consecutive ureteric stones done as a primary procedure: prospective outcomes from a University Hospital. Urol Int. 2019; 103:143-148.

19. Rice P, Prattley S, Somani BK.'Negative ureteroscopy' for stone disease: evidence from a systematic review. Curr Urol Rep. 2019; 20:13.

\section{Correspondence}

Miguel Eliseu, MD (Corresponding Author) mgl.nobre@gmail.com

Roberto Jarimba - rjarim-ba@gmail.com

Pedro Moreira - pedronetomoreira@gmail.com

Pedro Simões - pedrocorreiasimoes@gmail.com

Paulo Temido - ptemido@gmail.com

Arnaldo Figueiredo - ajcfigueiredo@gmail.com

Urology and Renal Transplantation Department, Coimbra Hospital and University Center

Praceta Professor Mota Pinto, 3004-561 Coimbra (Portugal) 